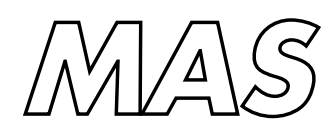

Modelling, Analysis and Simulation
Modelling, Analysis and Simulation
MAS Difference schemes for the class of singularly pertubed boundary value problems
I. Rafatov, S.N. Sklyar

Report MAS-R0310 SePtember 2003 
CWI is the National Research Institute for Mathematics and Computer Science. It is sponsored by the Netherlands Organization for Scientific Research (NWO).

CWI is a founding member of ERCIM, the European Research Consortium for Informatics and Mathematics.

CWI's research has a theme-oriented structure and is grouped into four clusters. Listed below are the names of the clusters and in parentheses their acronyms.

Probability, Networks and Algorithms (PNA)

Software Engineering (SEN)

Modelling, Analysis and Simulation (MAS)

Information Systems (INS)

Copyright (C) 2003, Stichting Centrum voor Wiskunde en Informatica

P.O. Box 94079, 1090 GB Amsterdam (NL)

Kruislaan 413, 1098 SJ Amsterdam (NL)

Telephone +31205929333

Telefax +31205924199

ISSN 1386-3703 


\title{
Difference schemes for the class of singularly perturbed boundary value problems
}

\begin{abstract}
This work deals with the construction of difference schemes for the numerical solution of singularly perturbed boundary value problems, which appear while solving heat transfer equations with spherical symmetry. The projective version of integral interpolation (PVIIM) method is used. Derived schemes allow to approximate the solution of the problem and the derivatives of the solution at the same time. Moreover, they allow to approximate the boundary conditions of general form in the framework of the same method. New schemes are tested in order to compare them with well known difference schemes. Estimates for rates of classical and uniform convergence are carried out.
\end{abstract}

2000 Mathematics Subject Classification: 65L12; 65L20; 34E15

Keywords and Phrases: Difference scheme, uniform convergence, singular perturbation 


\title{
Difference schemes for the class of singularly perturbed boundary value problems*
}

\author{
Ismail R. Rafatov ${ }^{1,2}$ and Sergey N. Sklyar ${ }^{2}$ \\ ${ }^{1}$ Centrum voor Wiskunde en Informatica, P.O. Box 94079, 1090 GB Amsterdam, The Netherlands \\ 2 American University - Central Asia, 205, Abdymomunova, 720000, Bishkek, Kyrgyzstan
}

\begin{abstract}
This work deals with the construction of difference schemes for the numerical solution of singularly perturbed boundary value problems, which appear while solving heat transfer equations with spherical symmetry. The projective version of integral interpolation (PVIIM) method is used. Derived schemes allow to approximate the solution of the problem and the derivatives of the solution at the same time. Moreover, they allow to approximate the boundary conditions of general form in the framework of the same method. New schemes are tested in order to compare them with well known difference schemes. Estimates for rates of classical and uniform convergence are carried out.
\end{abstract}

\section{Introduction}

Let us consider the following boundary value problem

$$
\left\{\begin{array}{l}
(\varepsilon / x)^{2}\left(x^{2} u^{\prime}\right)^{\prime}-q(x) u=f(x), \quad x \in(0,1) \\
u^{\prime}(0)=0, \quad \xi u(1)+\eta \varepsilon u^{\prime}(1)=\psi
\end{array}\right.
$$

We assume the functions $q, f$ in (1) to be sufficiently smooth, and additionally the conditions

$$
\begin{aligned}
& q(x) \geq q_{0}>0 \text { for } \quad x \in[0,1], \\
& \varepsilon \in(0,1], \quad \xi \geq 0, \quad \eta \geq 0, \quad \xi+\eta>0
\end{aligned}
$$

are satisfied.

Solving numerically similar problems (so called singularly perturbed problems) needs to use special difference schemes which guarantee the uniform convergence of the appropriate solution to the exact one [1]. There are two fundamental ways to construct the uniformly convergent numerical algorithms for singularly perturbed boundary problems. The first one uses the construction of the "special" difference schemes on uniform grids and starts from A.M.Ilyin's investigation [2]. The second one is based on the use of nonuniform grids adapted to the properties of the solution and is related to N.S.Bakhvalov's name historically [3]. In our opinion, the PVIIM [4], [5] may be considered as a combination of these two approaches. First, the method of the discretization keeps properties of the original differential problem automatically, therefore constructed schemes to be of the special type. Second, in the framework of the proposed method, an algorithm for the grid adaptation may be realized. Furthermore, the method allows to approximate the solution as well as its derivatives at the same time.

New difference schemes for problem (1), which converge uniformly with the first order in $\varepsilon$, were developed in [6] on the base of PVIIM. Our aim is the construction of the schemes of the second order of uniform in $\varepsilon$ accuracy on any irregular grid.

* This work has been presented at the International Conference NACoM-2003, Anglia Politechnic University, 23-26 May 2003, Cambridge, UK. Preprint is accepted for publication in the J. Applied Numerical Analysis \& Computational Mathematics. 
Throughout the paper we assume that the problem (1) has an unique solution from the class $\mathbf{C}^{1}[0,1] \cap \mathbf{C}^{2}(0,1)$. Let the operator $L$ of the problem (1) be defined by representations

$$
\left\{\begin{array}{l}
L v(0) \equiv-\varepsilon v^{\prime}(0) \\
L v(x) \equiv-(\varepsilon / x)^{2}\left(x^{2} v^{\prime}\right)^{\prime}+q(x) v, \quad x \in(0,1) \\
L v(1) \equiv \xi v(1)+\eta \varepsilon v^{\prime}(1)
\end{array}\right.
$$

for function $v$ from the above class. Using corresponding methods from [7] we can prove that $L$ is an operator of monotonic type. Hence, the following "comparison theorem" takes a place:

Lemma 1.1 Let us assume that the problem (1) satisfies the conditions (2), (3). Then the inequality $|L u(x)| \leq$ $|L v(x)|$ follows the inequality $|u(x)| \leq|v(x)|$ for any functions $u, v \in \mathbf{C}^{2}(0,1)(x \in[0,1])$.

The following statement guarantees a uniform boundedness (with respect to $\varepsilon$ ) of the solution of problem (1) (see [6]):

Lemma 1.2 Let us assume that the problem (1) satisfies the conditions (2), (3). Then its solution can be estimated by

$$
|u(x)| \leq \max _{0 \leq y \leq 1}|f(y)| / q_{0}+|\psi|\left(\xi+\eta q_{0} /\left(3+\sqrt{q_{0}}\right)\right)^{-1}
$$

for any $x \in[0,1]$.

\section{Difference schemes}

\subsection{An idea of the PVIIM}

Let us describe an idea of the PVIIM briefly. We introduce some grid $0=x_{1}<x_{2}<\ldots<x_{N}=1$ on the interval $[0,1]$ and denote $h_{i} \equiv x_{i+1}-x_{i},(i=1,2, \ldots, N-1), h \equiv \max _{1 \leq i \leq N-1}\left(h_{i}\right)$. Let $v^{h} \equiv\left\{v_{i}^{h}\right\}_{i=1}^{N}$ denote some grid function with the corresponding norm

$$
\left\|v^{h}\right\|_{h, \infty} \equiv \max _{1 \leq i \leq N}\left|v_{i}^{h}\right|
$$

Moreover, we denote $(v)^{h} \equiv\left\{v\left(x_{i}\right) \equiv v_{i}\right\}_{i=1}^{N}$ a projection of some continuous function $v(x)$ on the grid. Let constants $\bar{q}$ and $\bar{f}$ approximate functions $q(x)$ and $f(x)$ into the interval $\left[x_{i}, x_{i+1}\right]$. Multiplying equation (1) by $-x^{2} v(x)$, where $v(x)$ is a sufficiently smooth test function, then performing a partial integration of the result on $\left[x_{i}, x_{i+1}\right]$ and inserting values $\bar{q}$ and $\bar{f}$ in the integral identity, we obtain:

$$
\left\{\begin{array}{c}
{\left[-\varphi \varepsilon x^{2} v+u \varepsilon^{2} x^{2} v^{\prime}\right]_{x_{i}}^{x_{i+1}}+\int_{x_{i}}^{x_{i+1}} u\left[-\varepsilon^{2}\left(x^{2} v^{\prime}\right)^{\prime}+\bar{q} x^{2} v\right] d x=-\bar{f} \int_{x_{i}}^{x_{i+1}} x^{2} v d x} \\
+\delta\left(x_{i} x_{i+1},\right) \\
\delta\left(x_{i}, x_{i+1}\right) \equiv \int_{x_{i}}^{x_{i+1}}\{\bar{f}-f(x)+[\bar{q}-q(x)] u(x)\} x^{2} v d x .
\end{array}\right.
$$

Here we denote $\varphi(x) \equiv \varepsilon u^{\prime}(x)$. We choose testing functions $v^{(0)}(x)$ and $v^{(1)}(x)$ in identity (5) according to

$$
\begin{aligned}
& -\varepsilon^{2}\left(x^{2} v^{\prime}\right)^{\prime}+\bar{q} x^{2} v=0, \quad x \in\left(x_{i}, x_{i+1}\right) . \\
& \left\{\begin{aligned}
\left.x v^{(0)}\right|_{x=x_{i}}=1, & \left.x v^{(0)}\right|_{x=x_{i+1}}=0, \\
\left.x v^{(1)}\right|_{x=x_{i}}=0, & \left.x v^{(1)}\right|_{x=x_{i+1}}=1 .
\end{aligned}\right.
\end{aligned}
$$

Solution of the problems (6), (7) may be found easily:

$$
\begin{aligned}
& v^{(0)}(x)=\sinh \left(\left(x_{i+1}-x\right) \sqrt{q^{(0)}} / \varepsilon\right) /\left(x \sinh \left(h_{i} \sqrt{q^{(0)}} / \varepsilon\right)\right), \\
& v^{(1)}(x)=\sinh \left(\left(x-x_{i}\right) \sqrt{q^{(1)}} / \varepsilon\right) /\left(x \sinh \left(h_{i} \sqrt{q^{(1)}} / \varepsilon\right)\right) .
\end{aligned}
$$


Substituting $\bar{q}=q^{(0)}, \bar{f}=f^{(0)}$, and $v=v^{(0)}$ into (5), we have

$$
\begin{aligned}
& \varepsilon x_{i} \varphi\left(x_{i}\right)-\varepsilon^{2} x_{i+1}\left(u\left(x_{i+1}\right)-u\left(x_{i}\right)\right) / h_{i}+h_{i} q^{(0)}\left[\gamma\left(R^{(0)}\right) x_{i+1} u\left(x_{i+1}\right)\right. \\
& \left.+\mu\left(R^{(0)}\right) x_{i} u\left(x_{i}\right)\right]=-h_{i} f^{(0)}\left[\gamma\left(R^{(0)}\right) x_{i+1}+\mu\left(R^{(0)}\right) x_{i}\right]+\delta^{(0)}\left(x_{i}, x_{i+1}\right) .
\end{aligned}
$$

Analogously, for $\bar{q}=q^{(1)}, \bar{f}=f^{(1)}$, and $v=v^{(1)}$

$$
\begin{aligned}
& -\varepsilon x_{i+1} \varphi\left(x_{i+1}\right)+\varepsilon^{2} x_{i}\left(u\left(x_{i+1}\right)-u\left(x_{i}\right)\right) / h_{i}+h_{i} q^{(1)}\left[\mu\left(R^{(1)}\right) x_{i+1} u\left(x_{i+1}\right)\right. \\
& \left.+\gamma\left(R^{(1)}\right) x_{i} u\left(x_{i}\right)\right]=-h_{i} f^{(1)}\left[\mu\left(R^{(1)}\right) x_{i+1}+\gamma\left(R^{(1)}\right) x_{i}\right]+\delta^{(1)}\left(x_{i}, x_{i+1}\right) .
\end{aligned}
$$

Not taking into account the errors of approximation $\delta^{(0)}\left(x_{i,}, x_{i+1}\right)$ and $\delta^{(1)}\left(x_{i,}, x_{i+1}\right)$ in (9) and (10), after some transformations we obtain the following discrete problem, corresponding to (1):

$$
\left\{\begin{array}{l}
\varphi_{1}^{h}=0 \\
\varepsilon x_{i} \varphi_{i}^{h}-\varepsilon^{2} x_{i+1} D u_{i}^{h} \\
\left.+h_{i} q_{i}^{(0)}\left[\gamma\left(R_{i}^{(0)}\right) x_{i+1} u_{i+1}^{h}\right)+\mu\left(R_{i}^{(0)}\right) x_{i} u_{i}^{h}\right]=-f_{i}^{(0)} \sigma_{i}^{(0)} \\
-\varepsilon x_{i+1} \varphi_{i+1}^{h}+\varepsilon^{2} x_{i} D u_{i}^{h} \\
+h_{i} q_{i}^{(1)}\left[\mu\left(R_{i}^{(1)}\right) x_{i+1} u_{i+1}^{h}+\gamma\left(R_{i}^{(1)}\right) x_{i} u_{i}^{h}\right]=-f_{i}^{(1)} \sigma_{i}^{(1)} \\
\xi u_{N}^{h}+\eta \varphi_{N}^{h}=\psi . \quad(i=1,2, \ldots, N-1)
\end{array}\right.
$$

Here $u^{h} \equiv\left\{u_{i}^{h}\right\}_{i=1}^{N}$ and $\varphi^{h} \equiv\left\{\varphi_{i}^{h}\right\}_{i=1}^{N}$ approximate unknown grid functions $(u)^{h}$ and $(\varphi)^{h}$, respectively, and we denote

$$
\begin{gathered}
R_{i}^{(k)} \equiv h_{i} \sqrt{q_{i}^{(k)}} / \varepsilon, \quad k=0,1 \\
\mu(z)=(z \operatorname{coth} z-1) / z^{2}, \quad \gamma(z)=(1-z / \sinh z) / z^{2}
\end{gathered}
$$

and

$$
\begin{aligned}
D u_{i}^{h} & \equiv\left(u_{i+1}^{h}-u_{i}^{h}\right) / h_{i}, \sigma_{i}^{(0)} \equiv h_{i}\left[\gamma\left(R_{i}^{(0)}\right) x_{i+1}+\mu\left(R_{i}^{(0)}\right) x_{i}\right] \\
\sigma_{i}^{(1)} & \equiv h_{i}\left[\mu\left(R_{i}^{(1)}\right) x_{i+1}+\gamma\left(R_{i}^{(1)}\right) x_{i}\right], i=1,2, \ldots, N-1
\end{aligned}
$$

Excluding $\varphi_{i}^{h}(i=1,2, \ldots, N-1)$ from (11), we can rewrite the problem in the traditional third-point form

$$
\left\{\begin{array}{c}
L^{h} u_{1}^{h} \equiv-\varepsilon^{2} x_{2} D u_{1}^{h}+h_{1} q_{1}^{(0)}\left[\gamma\left(R_{1}^{(0)}\right) x_{2} u_{2}^{h}+\mu\left(R_{1}^{(0)}\right) x_{1} u_{1}^{h}\right] \\
=-f_{1}^{(0)} \sigma_{1}^{(0)} \\
L^{h} u_{i}^{h} \equiv-\varepsilon^{2} x_{i+1} D u_{i}^{h}+\varepsilon^{2} x_{i-1} D u_{i-1}^{h}+h_{i} q_{i}^{(0)}\left[\gamma\left(R_{i}^{(0)}\right) x_{i+1} u_{i+1}^{h}\right. \\
\left.+\mu\left(R_{i}^{(0)}\right) x_{i} u_{i}^{h}\right]+h_{i-1} q_{i-1}^{(1)}\left[\mu\left(R_{i-1}^{(1)}\right) x_{i} u_{i}^{h}+\gamma\left(R_{i-1}^{(1)}\right) x_{i-1} u_{i-1}^{h}\right] \\
=-f_{i}^{(0)} \sigma_{i}^{(0)}-f_{i-1}^{(1)} \sigma_{i-1}^{(1)},(i=2,3, \ldots, N-1) \\
L^{h} u_{N}^{h} \equiv \eta \varepsilon^{2} x_{N-1} D u_{N-1}^{h}+\eta h_{N-1}^{(1)} q_{N-1}\left[\mu\left(R_{N-1}^{(1)}\right) x_{N} u_{N}^{h}\right. \\
\left.+\gamma\left(R_{N-1}^{(1)}\right) x_{N-1} u_{N-1}^{h}\right]+\xi \varepsilon u_{N}^{h}=\varepsilon \psi-\eta f_{N-1}^{(1)} \sigma_{N-1}^{(1)}
\end{array}\right.
$$

The first and the last equations of (12) are the non-standard approximations of the boundary conditions of the problem (1). The family of the difference schemes (12) converges uniformly in $\varepsilon$ with the first order on any irregular grid (see [6]). For an example, consider the following variant of the choice of parameters $q_{i}^{(k)}$ and $f_{i}^{(k)}$ $(k=0,1 ; i=1,2, \ldots, N-1)$ in $(12)$ :

$$
\left\{\begin{array}{l}
q_{i}^{(0)}=q\left(x_{i}\right), \quad q_{i}^{(1)}=q\left(x_{i+1}\right), \\
f_{i}^{(0)}=f\left(x_{i}\right), \quad f_{i}^{(1)}=f\left(x_{i+1}\right) \quad(i=1,2, \ldots, N-1) .
\end{array}\right.
$$


In this case difference scheme (12) looks simple enough $\left(h_{i}=h, i=1,2, \ldots, N-1 ; \eta=0, \quad \xi=1\right)$ :

$$
\left\{\begin{array}{l}
h \sqrt{q(0)} /(\varepsilon \sinh (h \sqrt{q(0)} / \varepsilon)-h \sqrt{q(0)})\left(u_{1}^{h}-u_{2}^{h}\right)+u_{1}^{h}=-f(0) / q(0) \\
-1 / 4 \sinh ^{2}\left(h \sqrt{q\left(x_{i}\right)} / 2 \varepsilon\right)\left(x_{i+1} u_{i+1}^{h}-2 x_{i} u_{i}^{h}+x_{i-1} u_{i-1}^{h}\right)+x_{i} u_{i}^{h} \\
=-x_{i} f\left(x_{i}\right) / q\left(x_{i}\right), \quad(i=2,3, \ldots, N-1) \\
u_{N}^{h}=\psi
\end{array}\right.
$$

\subsection{Schemes of the second order of uniform accuracy}

Let us present one of the possible variants of the refinement of the scheme (12). We shall be limited by the choice of the parameters in the scheme (12) according with

$$
\left\{\begin{array}{l}
q_{i}^{(0)}=q_{i}^{(1)}=\left(q\left(x_{i}\right)+q\left(x_{i+1}\right)\right) / 2 \equiv q_{i+1 / 2}, \\
f_{i}^{(0)}=f_{i}^{(1)}=\left(f\left(x_{i}\right)+f\left(x_{i+1}\right)\right) / 2 \equiv f_{i+1 / 2}, \quad i=2,3, \ldots, N-1 .
\end{array}\right.
$$

In order to construct the scheme of the second order of uniform in $\varepsilon$ accuracy on any irregular grid, we need more precise then the estimation (4) of Lemma 1.2 information about asymptotic properties of the solution of the problem (1). We use the interpolative estimation, which is contained in the following statement:

Lemma 2.1 Let us assume, that the problem (1) satisfies conditions (2), (3) and $q, f \in \mathbf{C}^{1}[0,1]$. Then the estimation

$$
\begin{aligned}
& u(x)=x_{i} v_{i}^{(0)}(x) u_{i}+x_{i+1} v_{i}^{(1)}(x) u_{i+1}+ \\
& {\left[x_{i} v_{i}^{(0)}(x)+x_{i+1} v_{i}^{(1)}(x)-1\right] f_{i+1 / 2} / q_{i+1 / 2}+\Delta(x) \equiv u_{0}(x)+\Delta(x)}
\end{aligned}
$$

holds with

$$
|\Delta(x)| \leq 10 h / q_{0}
$$

on the interval $x \in\left[x_{i}, x_{i+1}\right](i=1,2, \ldots, N-1)$.

Functions $v_{i}^{(k)}(x)(k=0,1)$ in (15) are determined by formulas (8) and by the parameters $(14), u_{i} \equiv u\left(x_{i}\right)$ are the values of a grid function $(u)^{h}$.

Proof. To prove the representation (15) is possible by the help of formulas (9), (10) of the method of discretization. Putting the parameters $q_{i}^{(k)}$ and $f_{i}^{(k)}(k=1,2, i=2,3, \ldots, N-1)$ into (9) and (10) and excluding $\varphi(x)$, after some transformations we come to (15) with the error of approximation

$$
\begin{aligned}
\Delta(x)= & {\left[\delta^{(0)}\left(x, x_{i+1}\right)+\delta^{(1)}\left(x_{i}, x\right)\right] \sinh \left(\left(x-x_{i}\right) \sqrt{q_{i+1 / 2}} / \varepsilon\right) } \\
& \times \sinh \left(\left(x_{i+1}-x\right) \sqrt{q_{i+1 / 2}} / \varepsilon\right) /\left(\varepsilon x \sqrt{q_{i+1 / 2}} \sinh \left(h \sqrt{q_{i+1 / 2}} / \varepsilon\right)\right) .
\end{aligned}
$$

Skipping the proof of the inequality (16), which is technically bulky but theoretically simple enough, we finish the proof of the lemma.

By the virtue of (14) the following expression

$$
q_{i+1 / 2}-q(x)=\left(x_{i+1 / 2}-x\right) D(q)_{i}^{h}+\omega_{i}(x) q^{\prime \prime}\left(\rho_{i}\right),
$$

where

$$
\omega(x) \equiv\left(x_{i+1}-x\right)\left(x-x_{i}\right) / 2, x_{i+1 / 2} \equiv\left(x_{i}+x_{i+1}\right) / 2, \rho_{i} \in\left[x_{i}, x_{i+1}\right],
$$

and a similar representation for the $f(x)$ is valid for $x \in\left[x_{i}, x_{i+1}\right], i=1,2, \ldots, N-1$. Using (15) and (17), we can transform the local errors of approximation of the scheme (12) by separating the main asymptotic terms 
$(k=0,1):$

$$
\left\{\begin{aligned}
\delta^{(k)}\left(x_{i}, x_{i+1}\right)= & \int_{x_{i}}^{x_{i+1}}\left[D(f)_{i}^{h}+u_{0}(x) D(q)_{i}^{h}\right]\left(x_{i+1 / 2}-x\right) x^{2} v_{i}^{(k)}(x) d x \\
& +\bar{\delta}^{(k)}\left(x_{i}, x_{i+1}\right) \\
\bar{\delta}^{(k)}\left(x_{i}, x_{i+1}\right) \equiv & \int_{x_{i}}^{x_{i+1}}\left\{\left(x_{i+1 / 2}-x\right) \Delta(x) D(q)_{i}^{h}\right. \\
& \left.+\omega_{i}(x)\left[f^{\prime \prime}\left(\rho_{1, i}\right)+u(x) q^{\prime \prime}\left(\rho_{2, i}\right)\right]\right\} x^{2} v_{i}^{(k)}(x) d x
\end{aligned}\right.
$$

After calculating integrals in the first formulas of (18), we have:

$$
\left\{\begin{array}{c}
\delta^{(0)}\left(x_{i}, x_{i+1}\right)=h_{i}^{2} \mu\left(R_{i+1 / 2}\right) x_{i}\left[u_{i} D(q)_{i}^{h}+D(f)_{i}^{h}\right] / 4+T_{i}^{(0)} \\
+\bar{\delta}^{(0)}\left(x_{i}, x_{i+1}\right) \\
\delta^{(1)}\left(x_{i}, x_{i+1}\right)=-h_{i}^{2} \mu\left(R_{i+1 / 2}\right) x_{i+1}\left[u_{i+1} D(q)_{i}^{h}+D(f)_{i}^{h}\right] / 4+T_{i}^{(1)} \\
+\bar{\delta}^{(1)}\left(x_{i}, x_{i+1}\right)
\end{array}\right.
$$

The functionals

$$
\begin{aligned}
T_{i}^{(0)} \equiv & T_{i}^{(0)}[q, f] \equiv\left[(f / q)_{i+1 / 2} D(q)_{i}^{h}-D(f)_{i}^{h}\right]\left\{h _ { i } ^ { 2 } \left[2 \gamma\left(R_{i+1 / 2}\right) x_{i+1}\right.\right. \\
& \left.\left.-\mu\left(R_{i+1 / 2}\right) x_{i}\right] / 4+2 h_{i} \varepsilon^{2}\left[1 / 2-\gamma\left(R_{i+1 / 2}\right)-\mu\left(R_{i+1 / 2}\right)\right] / q_{i+1 / 2}\right\}, \\
T_{i}^{(1)} \equiv & T_{i}^{(1)}[q, f] \equiv\left[(f / q)_{i+1 / 2} D(q)_{i}^{h}-D(f)_{i}^{h}\right]\left\{h _ { i } ^ { 2 } \left[\mu\left(R_{i+1 / 2}\right) x_{i+1}\right.\right. \\
& \left.\left.-2 \gamma\left(R_{i+1 / 2}\right) x_{i}\right] / 4+2 h_{i} \varepsilon^{2}\left[\gamma\left(R_{i+1 / 2}\right)+\mu\left(R_{i+1 / 2}\right)-1 / 2\right] / q_{i+1 / 2}\right\}
\end{aligned}
$$

are introduced in (19) for the brevity of formulas.

Substituting all terms of $\delta^{(0)}$ with the exception of $\bar{\delta}^{(0)}$, and analogously, all terms of $\delta^{(1)}$ with the exception of $\bar{\delta}^{(1)}$ to the corresponding equation of the system (11), we arrive to the modified difference scheme:

$$
\left\{\begin{array}{l}
\varphi_{1}^{h}=0 \\
\varepsilon x_{i} \varphi_{i}^{h}-\varepsilon^{2} x_{i+1}\left(R_{i+1 / 2} / \sinh R_{i+1 / 2}\right) D u_{i}^{h}+S_{i}^{(0)}[q] u_{i}^{h} \\
=-S_{i}^{(0)}[f]+T_{i}^{(0)} \\
\quad \varepsilon x_{i+1} \varphi_{i+1}^{h}+\varepsilon^{2} x_{i}\left(R_{i+1 / 2} / \sinh R_{i+1 / 2}\right) D u_{i}^{h}+S_{i}^{(1)}[q] u_{i+1}^{h} \\
\quad=-S_{i}^{(1)}[f]+T_{i}^{(1)}, \quad(i=1,2, \ldots, N-1) \\
\xi u_{N}^{h}+\eta \varphi_{N}^{h}=\psi
\end{array}\right.
$$

Here

$$
\begin{aligned}
S_{i}^{(0)}[q] & \equiv h_{i}\left[\left(q_{i}+q_{i+1}\right) \gamma\left(R_{i+1 / 2}\right) x_{i+1} / 2+\left(3 q_{i}+q_{i+1}\right) \mu\left(R_{i+1 / 2}\right) x_{i} / 4\right] \\
S_{i}^{(1)}[q] & \equiv h_{i}\left[\left(q_{i}+3 q_{i+1}\right) \mu\left(R_{i+1 / 2}\right) x_{i+1} / 4+\left(q_{i}+q_{i+1}\right) \gamma\left(R_{i+1 / 2}\right) x_{i} / 4\right] .
\end{aligned}
$$

Excluding $\varphi_{i}^{h}(i=1,2, \ldots, N)$ from the system (20), we can obtain the discrete problem, related to the grid function $u^{h}$ :

$$
\left\{\begin{array}{c}
L^{h} u_{1}^{h} \equiv-\varepsilon^{2} x_{2}\left(R_{3 / 2} / \sinh R_{3 / 2}\right) D u_{1}^{h}+S_{1}^{(0)}[q] u_{1}^{h}=-S_{1}^{(0)}[f]+T_{1}^{(0)} \\
L^{h} u_{i}^{h} \equiv-\varepsilon^{2} x_{i+1}\left(R_{i+1 / 2} / \sinh R_{i+1 / 2}\right) D u_{i}^{h}+\left(S_{i}^{(0)}[q]+S_{i-1}^{(1)}[q]\right) u_{i}^{h} \\
+\varepsilon^{2} x_{i}\left(R_{i-1 / 2} / \sinh R_{i-1 / 2}\right) D u_{i-1}^{h} \\
=-\left(S_{i}^{(0)}[f]+S_{i-1}^{(1)}[f]\right)+T_{i}^{(0)}-T_{i-1}^{(1)}, \quad(i=2,3, \ldots, N-1) \\
L^{h} u_{N}^{h} \equiv \eta \varepsilon^{2} x_{N-1}\left(R_{N-1 / 2} / \sinh R_{N-1 / 2}\right) D u_{N-1}^{h}+ \\
\left(\eta S_{N-1}^{(1)}[q]+\xi \varepsilon\right) u_{N}^{h}=\varepsilon \psi-\eta S_{N-1}^{(1)}[f]+\eta T_{N-1}^{(1)}
\end{array}\right.
$$

The following statement contains the main result of this paper. 
Theorem 2.2 Let us assume that the problem (1) satisfies the conditions (2), (3) and $q, f \in \mathbf{C}^{2}[0,1]$. In this case the difference problem (20) has a unique solution, and for its solution $u^{h}$ and solution $u(x)$ of the problem (1) the estimate

$$
\left\|u^{h}-(u)^{h}\right\|_{h, \infty} \leq C h^{2}
$$

holds, where $C$ is a constant independent of $\varepsilon$ and $h$.

Pro of. Using the appropriate statement from [7] (or discrete principle of maximum from [8]) we can prove that the operator $L^{h}$ of the problem (21) is the operator of the monotone type for any parameters of the grid and $\varepsilon$. So, for the operator $L^{h}$ the discrete variant of the comparison theorem (see Lemma 1.1) is valid. The last statement guarantees an unambiguous resolvability of the problem (21) and shall be used for the proof of the estimate (22). Note that the grid function $w^{h} \equiv(u)^{h}-u^{h}$ satisfies the following system (see (19))

$$
\left\{\begin{array}{l}
L^{h} w_{1}^{h}=\bar{\delta}^{(0)}\left(x_{1}, x_{2}\right) \\
L^{h} w_{i}^{h}=\bar{\delta}^{(0)}\left(x_{i}, x_{i+1}\right)+\bar{\delta}^{(1)}\left(x_{i-1}, x_{i}\right), \quad(i=2,3, \ldots, N-1) \\
L^{h} w_{N}^{h}=\eta \bar{\delta}^{(1)}\left(x_{N-1}, x_{N}\right) .
\end{array}\right.
$$

Taking the estimations (4) and (16) into account, we have

$$
\left|\bar{\delta}^{(k)}\left(x_{i}, x_{i+1}\right)\right| \leq C h^{2} \sigma_{i}^{(k)}, \quad(i=1,2, \ldots, N-1, \quad k=0,1)
$$

where $C$ independent on $\varepsilon$ and $h$. By the virtue of definition of operator $L^{h}$ and functionals $S_{i}^{(k)}[q]$ the following estimations hold:

$$
\left\{\begin{array}{l}
L^{h}(1)_{1}^{h} \geq q_{0} \sigma_{1}^{(0)} \\
L^{h}(1)_{i}^{h} \geq q_{0}\left(\sigma_{i}^{(0)}+\sigma_{i-1}^{(1)}\right), \quad(i=2,3, \ldots, N-1) \\
L^{h}(1)_{N}^{h} \geq \eta q_{0} \sigma_{N-1}^{(0)} .
\end{array}\right.
$$

Comparing formulas (23), (24) and (25), we have

$$
\left|L^{h} w_{i}^{h}\right| \leq L^{h}\left(q_{0}^{-1} C h\right)_{i}^{h} \quad(i=1,2, \ldots, N) .
$$

The last expression, because of the comparison theorem, results in the inequality (22), thereby proving the theorem.

Note that the method for constructing of difference schemes, used in the this section, may be generalized for the system of equations [6].

\section{Numerical example}

The numerical experiments deal with the calculation of the orders of uniform and classical convergence according to the following algorithm (see also [1], [11]). Let $v_{\varepsilon}(x)$ be the solution of the differential problem (1), which depends on the parameter $\varepsilon$ and which is determined on the interval $[0,1]$. Let $v_{\varepsilon}^{h} \equiv\left\{v_{\varepsilon, i}^{h}\right\}_{i=1}^{N}$ be the grid function that approximates $v_{\varepsilon}\left(x_{i}\right)$ at the nodes of the uniform grid $x_{i}=(i-1) h(i=1,2, \ldots, N, N=1 / h+1)$ and is calculated for $h \in H \equiv\left\{h_{0} / 2^{j} \mid j=0,1, \ldots, k\right\}$ and $\varepsilon \in E \equiv\left\{\varepsilon_{0} / 2^{j} \mid j=0,1, \ldots, m\right\}$. Let us denote

$$
\delta(h, \varepsilon) \equiv\left\|v_{\varepsilon}^{h}-\left(v_{\varepsilon}\right)^{h}\right\|_{h, \infty}, \Delta(h) \equiv \Delta(h, v) \equiv \max _{\varepsilon \in E} \delta(h, \varepsilon) .
$$

The experimental orders of uniform and classical convergence (" $p$ " and " $\left.p_{0} "\right)$ are determined by the formulas

$$
p=\ln \left[1 / k \sum_{j=0}^{k-1}\left[\Delta\left(h_{0} / 2^{j}\right) / \Delta\left(h_{0} / 2^{j+1}\right)\right]\right] / \ln 2
$$




$$
p_{0}=\ln \left[1 / k \sum_{j=0}^{k-1}\left[\delta\left(h_{0} / 2^{j}, \varepsilon_{0}\right) / \delta\left(h_{0} / 2^{j+1}, \varepsilon_{0}\right)\right] / \ln 2\right]
$$

for values $h_{0}=1 / 8, \varepsilon_{0}=1 / 2, k=7, m=8$. Note that in the case of a piecewise constant $q(x)$ and $f(x)$, derived in the Section 2 schemes lead to the exact solution of the problem (1). Numerical experiments are done with coefficients

$$
\begin{aligned}
& q(x)=q_{0}+b_{0} x^{2}, \\
& f(x)=f_{0}+2 a_{0} \varepsilon^{2}\left(10 x^{2}-12 x+3\right)-a_{0} q_{0} x^{2}(1-x)^{2}-b_{0} x^{2} u_{0}(x)
\end{aligned}
$$

for $q_{0}=1, f_{0}=1, a_{0}=10, b_{0}=0.1, \xi=5, \psi=1, \eta=0$ (Dirichlet condition at the right boundary) and $\eta=0.5$ (mixed condition at the right boundary). Here $u_{0}(x)$ is the exact solution of the problem:

$$
\begin{aligned}
u_{0}(x)=-1 & +[6 /(5+0.5 \varepsilon[\operatorname{coth}(1 / \varepsilon) / \varepsilon-1])] \\
& \times[\sinh (x / \varepsilon) / x \sinh (1 / \varepsilon)]+10 x^{2}(1-x)^{2} .
\end{aligned}
$$

The quantities (26) and (27) are calculated for $v_{\varepsilon}(x) \equiv u(x)$ and $v_{\varepsilon}(x) \equiv \varphi(x)=\varepsilon u^{\prime}(x)$. Samarskii's well-known scheme [8] and scheme (11) with the approximations of $f_{i}^{(k)}$ and $q_{i}^{(k)}$ according with (13) and (14) are tested here. In the case of Samarskii's scheme, the boundary values of derivatives are calculated with the use of the directed difference (right point) and under the formula:

$$
u^{\prime}(0)=h(q(0) u(0)+f(0)) / 6 .
$$

Table1. The experimental orders of convergence(mixed condition)

\begin{tabular}{|l|c|c|c|c|}
\hline \multirow{2}{*}{ Method } & \multicolumn{2}{|c|}{$u(x)$} & \multicolumn{2}{c|}{$\varphi(x)$} \\
\cline { 2 - 5 } & $\begin{array}{c}\text { Uniform } \\
\text { convergence }\end{array}$ & $\begin{array}{c}\text { Classical } \\
\text { convergence }\end{array}$ & $\begin{array}{c}\text { Uniform } \\
\text { convergence }\end{array}$ & $\begin{array}{c}\text { Classical } \\
\text { convergence }\end{array}$ \\
\hline Samarskii $[8]$ & 0.30 & 1.10 & 0.23 & 0.98 \\
\hline$(11),(13)$ & 1.22 & 2.00 & 0.84 & 1.97 \\
\hline$(11),(14)$ & 1.06 & 1.99 & 0.99 & 1.98 \\
\hline
\end{tabular}

Table2. The experimental orders of convergence(Dirichlet condition)

\begin{tabular}{|l|c|c|c|c|}
\hline \multirow{2}{*}{ Method } & \multicolumn{2}{|c|}{$u(x)$} & \multicolumn{2}{c|}{$\varphi(x)$} \\
\cline { 2 - 5 } & $\begin{array}{c}\text { Uniform } \\
\text { convergence }\end{array}$ & $\begin{array}{c}\text { Classical } \\
\text { convergence }\end{array}$ & $\begin{array}{c}\text { Uniform } \\
\text { convergence }\end{array}$ & $\begin{array}{c}\text { Classical } \\
\text { convergence }\end{array}$ \\
\hline Samarskii $[8]$ & 0.46 & 1.98 & 0.21 & 0.98 \\
\hline$(11),(13)$ & 1.04 & 2.00 & 0.61 & 1.97 \\
\hline$(11),(14)$ & 0.87 & 2.00 & 0.87 & 1.98 \\
\hline
\end{tabular}

The analysis of tables allows to conclude, that the numerical experiment confirms the statement about a uniform convergence (with the first order) of the solution of the difference problem (11) to the solution of the initial continuous problem (1). Moreover, as a result of this experiment, the hypothesis about a uniform convergence (with the first order) of fluxes may be formulated.

\section{References}

[1] Doolan E.P.H., Miller J.J., Scilders W.H.A., Uniform numerical methods for problems with initial and boundary layers (Boole Press, Dublin, 1980).

[2] Ilyin A.M., Matem.Zametki 6, 237-248 (1969).

[3] Bakhvalov N.S., J.Comput.Math.Math.Phys. 9, 842-859 (1969).

[4] Sklyar S.N., in: Proceedings of the International Conference AMCA-95, Novosibirsk, 1995, edited by A.S.Alekseev and N.S.Bakhvalov (NCC Pablisher, Novosibirsk, 1995), p.380.

[5] Sklyar S.N. and Bakirov J.J., Izvesia. Acad. Nauk Kyrgyz. 2-3, 36-47 (1997). 
[6] Sklyar S.N. and Rafatov I.R., J.Comp.Math.Math.Phys. 42, 1397-1407 (2002).

[7] Collatz L., Functional analysis and numerical mathematics (Mir, Moscow, 1969).

[8] Samarskiy A.A., The theory of difference schemes (Nauka, Moscow, 1983).

[9] Samarskiy A.A. and Nikolaev E.S., Numerical methods for grid equatios (Nauka, Moscow, 1978).

[10] Ortega J. and Rheinbold W., Iterative solution of nonlinear equations in several variables (Mir, Moscow, 1975).

[11] Farell P.A., IMA J.Numer.Anal. 7, 459-472 (1987). 